\title{
pygtc: beautiful parameter covariance plots (aka. Giant Triangle Confusograms)
}

\section{DOI: $10.21105 /$ joss.00046}

\section{Software}

- Review ¿

- Repository ca

- Archive ¿

\section{Licence}

Authors of JOSS papers retain copyright and release the work under a Creative Commons Attribution 4.0 International License (CC-BY).

\section{Sebastian Bocquet ${ }^{1}$ and Faustin W. Carter $^{1}$}

1 Argonne National Laboratory

\section{Summary}

Multi-dimensional model parameter spaces are commonly sampled using Markov Chain Monte-Carlo (MCMC) methods or more advanced algorithms as implemented, for instance, in emcee (Foreman-Mackey et al. 2013) or PyMultiNest (Buchner et al. 2014). The recovered parameter constraints are usually displayed on a grid in which the diagonal shows the 1-dimensional posteriors and the lower-left half shows the pairwise projections. Due to the triangular appearance, such plots are typically referred to as "triangle" plots. If the parameter space is large, the resulting plot can be visually overwhelming; we refer to such a figure as a Giant Triangle Confusogram (GTC).

Several packages exist to produce these plots and range in functionality from the lightweight corner (Foreman-Mackey 2016), to the feature-heavy getdist (Lewis 2015), which allows arbitrary large data sets and contains nearly every conceivable option and customization (at the expense of a steep learning curve). pygtc aims to fill the parameter space in between by producing beautiful publication-ready figures with as little as a single line of code, while allowing for an arbitrary number of sets of likelihood surfaces (a crucial feature in our field of cosmology). Various user-friendly options allow for further adjustments and ensure that details such as font sizes are chosen to match the layout of several astrophysical journals.

Figure 1 shows an example figure (comparing three sets of data from different analyses) that was produced with the pygtc package and formatted for the Astrophysical Journal, all with the following line of code:

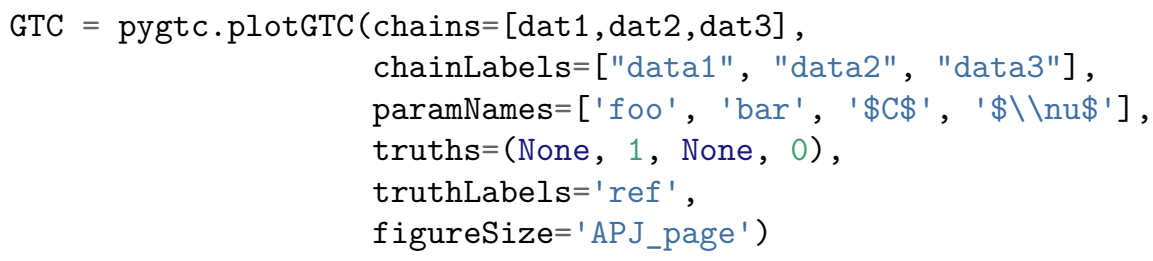

pygtc relies on numpy (Walt, Colbert, and Varoquaux 2011), matplotlib (Hunter 2007), and scipy (Jones et al. 2001-2001--) (optional) to produce aesthetically appealing GTCs, and is designed to accept the standard output of the emcee package or any other likelihood sampler that returns a list of (weighted or unit-weighted) sample points. The source code for the latest release of pygtc is available at Zenodo (Bocquet and Carter 2016b) and development is ongoing at GitHub (Bocquet and Carter 2016a).

\section{Acknowledgements}

Work at Argonne National Laboratory was supported under the U.S. Department of Energy contract DE-AC02-06CH11357. 


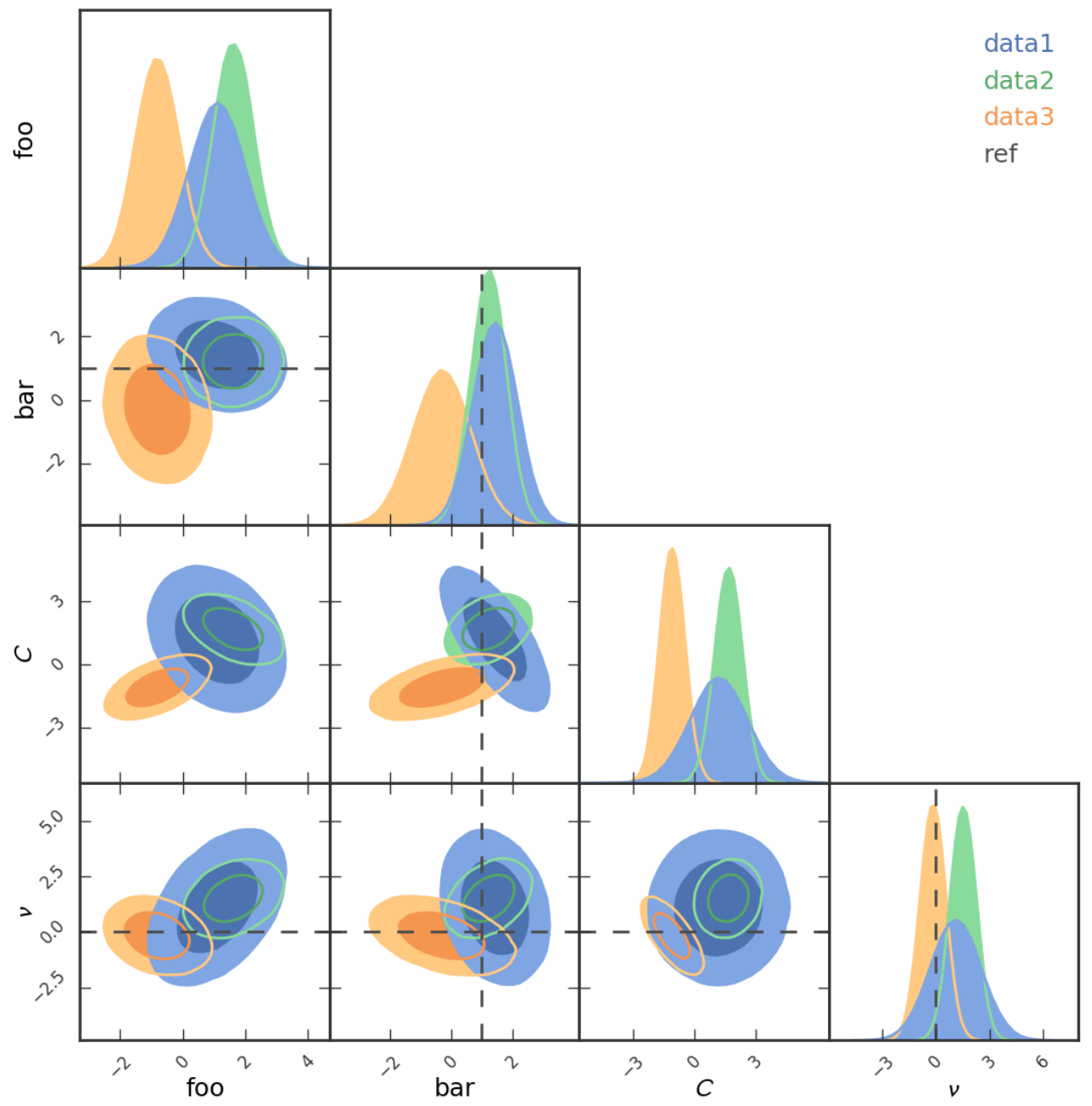

Figure 1: A GTC produced by pygtc. 


\section{References}

Bocquet, Sebastian, and Faustin W. Carter. 2016a. "Pygtc Github Repository." https: //github.com/SebastianBocquet/pygtc.

2016b. "Pygtc Zenodo Archive." doi:10.5281/zenodo.159091.

Buchner, J., A. Georgakakis, K. Nandra, L. Hsu, C. Rangel, M. Brightman, A. Merloni, M. Salvato, J. Donley, and D. Kocevski. 2014. "X-ray spectral modelling of the AGN obscuring region in the CDFS: Bayesian model selection and catalogue" 564 (April): A125. doi:10.1051/0004-6361/201322971.

Foreman-Mackey, Daniel. 2016. "Corner.py: Scatterplot Matrices in Python." The Journal of Open Source Software 24. doi:10.21105/joss.00024.

Foreman-Mackey, Daniel, David W. Hogg, Dustin Lang, and Jonathan Goodman. 2013. "Emcee: The Mcmc Hammer." Publications of the Astronomical Society of the Pacific 125 (925): 306. http://stacks.iop.org/1538-3873/125/i=925/a=306.

Hunter, J. D. 2007. "Matplotlib: A 2d Graphics Environment." Computing in Science \&ु Engineering 9 (3). IEEE COMPUTER SOC: 90-95. doi:10.1109/MCSE.2007.55.

Jones, Eric, Travis Oliphant, Pearu Peterson, and others. 2001-2001--. "SciPy: Open Source Scientific Tools for Python." doi:10.1109/MCSE.2011.37.

Lewis, Antony. 2015. "Getdist Github Repository." https://github.com/cmbant/getdist.

Walt, Stéfan van der, S. Chris Colbert, and Gaël Varoquaux. 2011. "The Numpy Array: A Structure for Efficient Numerical Computation." Computing in Science 8 Engineering 13 (2): 22-30. doi:http://dx.doi.org/10.1109/MCSE.2011.37. 